\title{
Growth rate as a modulator of tooth patterning during adaptive radiations
}

\author{
Authors: Alexa Sadier ${ }^{1, *}$, Neal Anthwal ${ }^{1,2}$, Andrew L. Krause ${ }^{3}$, Renaud Dessalles ${ }^{1,4}$, Michael \\ Lake $^{5}$, Laurent Bentolila ${ }^{5}$, Robert Haase ${ }^{6}$, Natalie Nieves ${ }^{1}$, Sharlene Santana ${ }^{7}$ and Karen Sears ${ }^{1, *}$ \\ Affiliations: \\ ${ }^{1}$ Department of Ecology and Evolutionary Biology, University of California Los Angeles, USA \\ ${ }^{2}$ Centre for Craniofacial and Regenerative Biology, King's College London, UK \\ ${ }^{3}$ Mathematical Institute, University of Oxford, UK \\ ${ }^{4}$ Greenshield, 46 rue Saint-Antoine, 75004 PARIS, France \\ ${ }^{5}$ CNSI, ALMS, University of California Los Angeles, USA \\ ${ }^{6}$ DFG Cluster of Excellence "Physics of Life", TU Dresden, Germany \\ ${ }^{7}$ Department of Biology and Burke Museum of Natural History and Culture, University of \\ Washington, Seattle, WA,USA \\ *Corresponding authors. Emails: asadier@ucla.edu, ksears@ucla.edu
}

The discovery of mechanistic rules that underlie phenotypic variation has been a longstanding goal of evolutionary biology. Developmental processes offer a potential source for such rules because they translate genomic variation into the population-scale phenotypic variation. However, our understanding of developmental rules is based on a handful of well-established model species which hindered identifying rules and investigating their evolution. Recent methodological advances, such as $\mu \mathrm{CT}$ scanning on soft tissues, two-photon imaging and modelling have facilitated the study of how developmental processes shape phenotypic variation in diverse, non-traditional model species. Here, we use the outstanding dental diversity of bats to investigate how the interplay between developmental processes can explain the morphological diversity in teeth. We find that the inhibitory cascade model, which has been used to predict the proportions of teeth and other serial organs, poorly predicts the variation in tooth number and size in bats. Instead, by tinkering with reaction/diffusion processes, we identify jaw growth as a key driver of the phenotypic evolution of tooth number and size critical to the different diets. By studying developmental processes in the context of adaptive evolution, we are able to discover a new developmental rule that explain and predict interspecific variation in serial organ number and proportion.

The search for general rules that govern the evolution of organismal forms has been a main goal of evolutionary developmental biology (1-4). The models pioneered by Alan Turing, David Raup, Lewis Wolpert and others have suggested that developmental constraints influence the evolution of organisms by biasing, i.e. constraining or facilitating the evolution of shape toward particular phenotypic outcomes (4-6). In the last 15 years, the increasing development of mathematical modelling, the renewed interest for Turing mechanisms and the study of developmental bias have reinvigorated the interest in defining development-based rules that generate many patterns observed in nature (e.g. teeth, hair, vertebrae) $(2-4,7)$. Perhaps one of the most iconic of these rules is the "inhibitory cascade" (IC) which proposes an activator/inhibitor system that successfully predicts the variation in size, proportion (i.e., relative sizes) and sequential formation of serially repeated structures within the body $(2,3)$. However, the exploration of more clades, species and organs in detail has revealed that this rule is not 
universal and fails to reproduce the inter- or intraspecific variation among species (8-12), highlighting the need to re-examine how developmental mechanisms themselves evolve and interact to fully understand the developmental foundations of phenotypic diversification.

To study this question, we need both a model organ for which such rules are well established, and a clade that exhibits a tremendous morphological variation of this organ. We chose noctilionoid bats teeth, as this system possesses these characteristics. Emerging forty-five million years ago, this clade underwent a major adaptive radiation such that today more than 200 species utilize nearly all possible mammalian diets (i.e., fruit, nectar and pollen, leaves, seeds, arthropods, small vertebrates, fish, and even blood). Like the evolution of disparate, diet-related beak shapes in Darwin's finches, noctilionoid bats evolved a wide diversity of skull shapes to fill with these dietary niches (13). In association with this wide variety of ecological niches, noctilionoid bats exhibit a tremendous diversity in tooth proportion, size, morphology, and number (Fig. 1, S1 and S2) (14) of their complete mammalian dentition (i.e. incisors, canine, premolars and molars): the number of premolars ranges from two to three (P2, P3 and P4, P3 and $\mathrm{P} 4$ presenting decidual teeth $\mathrm{dP} 3$ and $\mathrm{dP} 4)$ and the number of molars from one to three (M1, M2 and M3) (14-17). In addition, tooth proportions and shape are highly variable with gains and losses happening multiple times in evolution. Finally, in association with this variation in tooth number and size, their snout and jaw length is highly diverse, with snouts and jaws extremely elongated in nectarivorous forms, and highly shortened in durophagous frugivores, which has been associated with variation in cell division during development and heterochronies (17-19).

Using the outstanding variation of bat teeth as a natural experiment, we explore the developmental rules that pattern serial structures and how these rules have evolved to produce adaptive morphological variation. We reveal that bat premolars and molars are formed by distinct signaling cascades and that growth, by perturbing underlying Turing processes, acts as a simple developmental rule that modulates the number and size of the different classes of teeth and possibly other ectodermal appendages. Finally, we propose that modulating growth could explain the rapid evolution of repeated structures number and size in the context of adaptive radiations.

We first tested if the known-IC rule accurately describes the developmental processes that shape postcanine tooth proportions in noctilionoids. If this is the case, tooth $(\mathrm{T})$ proportions should follow the relationship $T(n+2) / T(n)=2(T(n+1) / T(n)-1(2)$, $n$ being the first tooth to develop. Instead, we found that only $63.7 \%$ of species molar proportions and $8 \%$ of species premolar proportions fell inside the predicted IC morphospace (Fig. 1) showing that the IC rule is not sufficient to predict the variation observed in these bats. To exclude that both tooth classes originate from a single signaling center (either the P4 of the M1, Fig. 1A) that would initiate the same cascade on both sides, we tested if the P4-M1-M2 triplet follows proportions predicted by the inhibitory cascade (Fig. S3) as done previously (20) and studied tooth development in eight species of noctilinoid bats that encompass much of the diversity in tooth number and size using micro-computed tomography $(\mu \mathrm{CT})$. We found that more than half of the species proportions deviate from the model according to the P4-M1-M2 triplet (Fig. S3). Premolars and molars form from two distinct buds (Fig. 2A and Fig. S6) appearing at the same developmental stage (Carnegie Stage 19, CS19) initiating two independent cascades (Fig. 2A) as the dental lamina grows in both directions (Fig. 2A); a finding consistent with molar and premolar development 
being largely independent, in contrast to what has been suggested but never verified developmentally in other mammals (20).

To explore the underlying developmental mechanisms of their formation (2), we quantified morphological variation in the premolars and molars across 118 species $(\mathrm{N} \leq 3)$ of noctilionoid bat spanning all dietary guilds. Species were classified into four main morphogroups (see supplementary data regarding other morphogroups, Fig S1, S2 and S4, Tables S1 and 3) based on relative tooth number, tooth size, and jaw length (Fig. 1, Fig S1). Some of these groups, such as 2P3MR, are polyphyletic and record independent events of tooth losses in noctilionoid bats. We found that the number of teeth is linked to the size of the jaw among species (Fig. S5), sometimes convergently, and that tooth proportions become more disparate in size among species with shorter jaws that exhibit less equal teeth (typically frugivores; Fig. S4) suggesting that the process of jaw elongation plays a role in the generation of tooth proportions and number.

To further explore the mechanisms linking tooth number and size with jaw length variation, we studied if the sequence of premolar and molar appearance during development depends on the available dental lamina space (Fig. 2A). In 3P3MR bats, we observed that the dP4 development is followed by the sequential development of dP3 and P2 (Fig. 2A, 3A and B) which is consistent with what is seen in the classical IC model. In 2P3MR bats which have a slightly smaller jaw, further examination of the dental lamina revealed that, while the dP3 forms and mineralizes, its replacement, adult P3 is initiated but does not develop further (Fig. 2B). On the contrary, in 3P3ML bats that exhibit an elongated jaw, dP3 and P2 appear almost simultaneously as the jaw develops rapidly (Fig. 3A and B). Together, these results suggest that the development and timing of formation of the different premolar buds is influenced by the space available in the jaw, likely because new teeth are able to form only at a certain distance from each other as predicted by the IC (2). This finding is supported by the pattern of tooth formation in the shorterjawed 2P2MS and 2P3MS bats; the development of the dP3 is initiated but the incipient tooth fails to grow and/or mineralize resulting in the loss of both $\mathrm{dP} 3$ and P3 (Fig. 2B and S6). In 2P2MS bats, such as A. phaeotis, the M3 is lost with no evidence that it ever started to develop (Fig. S6). These patterns of loss are consistent with the variation observed in adult bats: the smallest teeth, specifically the middle premolar (P) 3 and the last molar (M) 3, exhibit the most variation in size among species with reduced jaw lengths (Fig. S4); P2 and P4 and M1 and M2, respectively, exhibit similar size variability among species with reduced and unreduced jaw lengths. Of note, the loss of the M3 is polymorphic in species of short-faced bats that exhibit variation in size $(21,22)$, which is consistent with the presence/absence of M3 being dependent on the available space in the developing jaw (A. watsoni being just at the limit condition for which the M3 develops further or not). To sum up, premolars and molars exhibit very different patterns of tooth loss, with premolars losing the middle tooth gradually (dP3 and then P3) and molars losing the last tooth of the row, M3.

The results presented above support the hypothesis that the number and proportions of premolar and molar teeth are linked to the size of the jaw and, in particular, how fast the jaw is growing and where that growth is located along the jaw at the time of tooth bud formation. To explore this idea, we measured the growth rate of the premolar area during development in 3D models reconstructed from phosophotungstic acid contrasted $\mu \mathrm{CT}$ scans in eight focal species representative of our morphogroups. These measures revealed that species with a regular 
(average) jaw (3P3MR and and 2P3MR) exhibit a moderate peak of growth around stage 20 as dP3 and P2 develop (Fig. 3A and B). In 3P3ML bats with an elongated jaw, this peak is 3 times faster than of regular jaws and correspond to the almost simultaneous formation of $\mathrm{dP} 3$ and $\mathrm{P} 2$. On the contrary, in short-faced bats (2P3MS and 2P2MS), no growth peak is seen, the growth rate being overall lower than for other bats in this region. To examine these patterns in more detail, we used EdU and PCNA labeling to trace cell proliferation during the development of the jaw (Fig. 3C and D, Fig. S7). 3P3ML bats exhibit a higher rate of cell divisions in the premolar area compared to the others, while $2 \mathrm{P} 3 \mathrm{MS}$ and $2 \mathrm{P} 2 \mathrm{MS}$ bats show a lower number of dividing cells. These results echoes other results in nectar feeder phyllostomids (18) and confirm the idea that differences in cell proliferation rate drive craniofacial differences between species (19).

These findings suggest that the growth rate of the jaw might perturb both the sequence apparition of teeth and the reaction/diffusion mechanisms that shape their proportions. As these two parameters depend on the activation/inhibition processes that pattern repeated structures, we postulated that the rapid variation in tooth number and size observed in noctilionoid bats can be simply explained by the perturbations induced by the variation in growth rate in the Turing mechanisms behind the ICs. We computationally tested if we were able to reproduce the various phenotypes observed during development by modelling variation in jaw size and growth. We implemented a simple model of a Turing-type reaction-diffusion system (Document S1) to recapitulate the different sequences of insertions observed (Fig. $4 \mathrm{~A}$ and B). Using only apical growth, we successfully recapitulated two of the four morphogroups (corresponding to 3P3MR P. quadridens and 3P3ML M. redmani) (Fig. 4A). Presupposing exogenous spatial gradients (plausibly corresponding to a pre-pattern or additional spatial modulation), we also captured the insertion sequence and qualitatively the size variations observed in $2 \mathrm{P} 3 \mathrm{MS}$ A. jamaicensis (Fig. 4B). These results demonstrate that while there are undoubtedly others factors influencing the insertion and modulation of teeth signaling centers (that could explain the 3P2M group that lose a molar while have a long jaw with diastemas; Fig. S2, S4 and 5), simple models of reactiondiffusion and differential growth are sufficient to explain much of the observed variation, confirming the importance of growth rate and jaw length variation in modulating tooth number and size.

In our study, we identified multiple factors to explain the rapid gain/loss of teeth as well as their diversity in noctilionoid bats, will have multiple implications for organ evolution. First, we showed that growth, by perturbing the IC at the origin of tooth patterning, acts as a simple modulator of tooth number and size. As both the IC cascade and growth are implicated in the development of ectodermal appendages in general, these conclusions are likely to extend to other ectodermal organs and propose a testable model to explain how their number and proportions can rapidly evolve, simply by modulating growth rate. This finding is consistent with what was suggested in other ectodermal appendages, such as palatal rugae, in which a growth burst drives the apparition of new segments in the resulting available space in mice and hamster $(23,24)$, or other ectodermal organs $(25,26)$ that follow Turing pattern formation without a directional growth. Second, our results shed light on the origin of the different tooth classes by revealing that premolar and molars are patterned by two different cascades. Up to now, the origin of different tooth classes has been suggested to be driven by an early "hox code" based on early differences in gene expression pattern in presumptive territories for the apparition of the different tooth classes (27), but the differences between the difference tooth classes remained obscure 
(28). Our work, by showing that premolars and molars develop independently with two different dynamics, uncover another mechanism that could explain the differences between the different tooth classes that could act in tandem with the hox code. More largely, by looking at the difference of patterning between two closely related organ classes, our results constitute an example of how developmental mechanisms diverge during organ diversification.

More widely, our work demonstrates how new morphologies can be reached by changing the interaction between multiple developmental constraints during the burst of diversity that accompanies adaptive radiations. While studies of developmental bias often solely focus on the evolution on one constraint to explain the evolution of characters, our work, by showing an example of how predicted constraints are evaded multiple times in two different tooth classes (Fig. 4C), reveals the importance of studying the complex interaction of different developmental rules to fully understand the evolution of new morphologies during the colonization of new ecological niches.

\section{References}

1. A. P. Moczek, K. E. Sears, A. Stollewerk, P. J. Wittkopp, P. Diggle, I. Dworkin, C. Ledon-Rettig, D. Q. Matus, S. Roth, E. Abouheif, F. D. Brown, C.-H. Chiu, C. S. Cohen, A. W. D. Tomaso, S. F. Gilbert, B. Hall, A. C. Love, D. C. Lyons, T. J. Sanger, J. Smith, C. Specht, M. Vallejo-Marin, C. G. Extavour, The significance and scope of evolutionary developmental biology: a vision for the 21 st century. Evol. Dev. 17, 198-219 (2015).

2. K. D. Kavanagh, A. R. Evans, J. Jernvall, Predicting evolutionary patterns of mammalian teeth from development. Nature. 449, 427-432 (2007).

3. N. M. Young, B. Winslow, S. Takkellapati, K. Kavanagh, Shared rules of development predict patterns of evolution in vertebrate segmentation. Nat. Commun. 6, 6690 (2015).

4. S. Kondo, T. Miura, Reaction-Diffusion Model as a Framework for Understanding Biological Pattern Formation. Science. 329, 1616-1620 (2010).

5. J. M. Smith, R. Burian, S. Kauffman, P. Alberch, J. Campbell, B. Goodwin, R. Lande, D. Raup, L. Wolpert, Developmental Constraints and Evolution: A Perspective from the Mountain Lake Conference on Development and Evolution. Q. Rev. Biol. 60, 265-287 (1985).

6. D. M. Raup, Geometric Analysis of Shell Coiling: General Problems. J. Paleontol. 40, 1178-1190 (1966).

7. P. D. Polly, Development with a bite. Nature. 449, 413-414 (2007).

8. C. C. Roseman, L. K. Delezene, The Inhibitory Cascade Model is Not a Good Predictor of Molar Size Covariation. Evol. Biol. 46, 229-238 (2019).

9. N. S. Vitek, C. C. Roseman, J. I. Bloch, Mammal Molar Size Ratios and the Inhibitory Cascade at the Intraspecific Scale. Integr. Org. Biol. 2 (2020), doi:10.1093/iob/obaa020.

10. L. J. Hlusko, C. A. Schmitt, T. A. Monson, M. F. Brasil, M. C. Mahaney, The integration of quantitative genetics, paleontology, and neontology reveals genetic underpinnings of primate dental evolution. Proc. Natl. Acad. Sci. 113, 9262-9267 (2016).

11. K. E. Carter, S. Worthington, The evolution of anthropoid molar proportions. BMC Evol. Biol. 16 (2016), doi:10.1186/s12862-016-0673-5.

12. G. B. Grant PR, How and why species multiply - the radiation of Darwin's finches. Princet. NJ USA Princet. Univ. Press (2008). 
13. L. M. Dávalos, M. A. R. Mello, Eds., Phyllostomid Bats: A Unique Mammalian Radiation (University of Chicago Press, Chicago, IL, 2020; https://press.uchicago.edu/ucp/books/book/chicago/P/bo50700096.html).

14. P. W. Freeman, Form, Function, and Evolution in Skulls and Teeth of Bats (Bat Biology and Conservation: Smithsonian Institution Press, 1998).

15. P. W. Freeman, Frugivorous and animalivorous bats (Microchiroptera): dental and cranial adaptations. Biol. J. Linn. Soc. 33, 249-272 (1988).

16. P. W. Freeman, Nectarivorous feeding mechanisms in bats, 26 (1995).

17. S. E. Santana, S. Strait, E. R. Dumont, The better to eat you with: functional correlates of tooth structure in bats. Funct. Ecol. 25, 839-847 (2011).

18. J. Camacho, A. Heyde, B.-A. S. Bhullar, D. Haelewaters, N. B. Simmons, A. Abzhanov, Peramorphosis, an evolutionary developmental mechanism in neotropical bat skull diversity. Dev. Dyn. 248, 1129-1143 (2019).

19. J. Camacho, R. Moon, S. K. Smith, J. D. Lin, C. Randolph, J. J. Rasweiler, R. R. Behringer, A. Abzhanov, Differential cellular proliferation underlies heterochronic generation of cranial diversity in phyllostomid bats. EvoDevo. 11, 1-17 (2020).

20. A. R. Evans, E. S. Daly, K. K. Catlett, K. S. Paul, S. J. King, M. M. Skinner, H. P. Nesse, J. J. Hublin, G. C. Townsend, G. T. Schwartz, J. Jernvall, A simple rule governs the evolution and development of hominin tooth size. Nature. 530, 477-80 (2016).

21. G. Chaverri, O. E. Quirós, T. H. Kunz, Ecological Correlates of Range Size in the TentMaking Bat Artibeus watsoni. J. Mammal. 88, 477-486 (2007).

22. C. W. Dick, Variation in the Dental Formula of the Ipanema Bat, Pygoderma bilabiatum. Southwest. Nat. 47, 505-508 (2002).

23. S. Pantalacci, M. Sémon, A. Martin, P. Chevret, V. Laudet, Heterochronic shifts explain variations in a sequentially developing repeated pattern: palatal ridges of muroid rodents. Evol. Dev. 11, 422-433 (2009).

24. S. Pantalacci, J. Prochazka, A. Martin, M. Rothova, A. Lambert, L. Bernard, C. Charles, L. Viriot, R. Peterkova, V. Laudet, Patterning of palatal rugae through sequential addition reveals an anterior/posterior boundary in palatal development. BMC Dev. Biol. 8, 116 (2008).

25. S. Sick, S. Reinker, J. Timmer, T. Schlake, WNT and DKK Determine Hair Follicle Spacing Through a Reaction-Diffusion Mechanism. Science. 314, 1447-1450 (2006).

26. C. Mou, B. Jackson, P. Schneider, P. A. Overbeek, D. J. Headon, Generation of the primary hair follicle pattern. Proc. Natl. Acad. Sci. U. S. A. 103, 9075-9080 (2006).

27. J. Catón, A. S. Tucker, Current knowledge of tooth development: patterning and mineralization of the murine dentition. J. Anat. 214, 502-515 (2009).

28. A. Sadier, W. R. Jackman, V. Laudet, Y. Gibert, The Vertebrate Tooth Row: Is It Initiated by a Single Organizing Tooth? BioEssays. 42, 1900229 (2020).

29. C. A. Mancina, Pteronotus macleayii. Mamm. Species, 1-3 (2005).

30. A. Moreno-Valdez, R. L. Honeycutt, W. E. Grant, Colony Dynamics of Leptonycteris nivalis (Mexican Long-Nosed Bat) Related to Flowering Agave in Northern Mexico. J. Mammal. 85, 453-459 (2004).

31. P. M. Velazco, A. L. Gardner, B. D. Patterson, Systematics of the Platyrrhinus helleri species complex (Chiroptera: Phyllostomidae), with descriptions of two new species: SYSTEMATICS OF PLATYRRHINUS HELLERI. Zool. J. Linn. Soc. 159, 785-812 (2010).

32. M. Schwarzkopf, M. C. Liu, S. J. Schulte, R. Ives, N. Husain, H. M. T. Choi, N. A. Pierce, "Hybridization chain reaction enables a unified approach to multiplexed, quantitative, 
high-resolution immunohistochemistry and in situ hybridization" (2021), p. 2021.06.02.446311, , doi:10.1101/2021.06.02.446311.

33. H. Hama, H. Hioki, K. Namiki, T. Hoshida, H. Kurokawa, F. Ishidate, T. Kaneko, T. Akagi, T. Saito, T. Saido, A. Miyawaki, Sca 1 eS: an optical clearing palette for biological imaging. Nat. Neurosci. 18, 1518-1529 (2015).

\section{Acknowledgments}

We specially thank A. Couzens, A. Tucker and V. Laudet for their critical reading of the manuscript. We thank the CNSI and ALMS imaging centers for providing assistance for imaging through their core facilities and Leilei Yin from X-ray imaging facility at UIUC. We thank N. Simmons and B. Felton for organizing the fieldtrip in Belize, L. Rostant in Trinidad, M. Santiago and J. Almonthe in Dominican Republic and A. Rodrigues in Puerto Rico for their support in the acquisition of permits for sampling and sample exportation. We thank Nicolas Rochette for his help with statistical analysis.

\section{Funding sources}

University of California, Los Angeles.

National Science Foundation (NSF) IOS grant Award 2017803.

\section{Author contributions:}

Conceptualization: A.S. and K.S. Methodology: A.S. and K.S. Formal analysis: A.S. Investigation: A.S. Data collection: A.S., N.N., S.S., and K.S. Modeling: A.K. and R.D. Supervision: A.S and K.S. Imaging: A.S, M.L. (core facility) and L.B. (core facility). Image processing: A.S. and R.H. Writing - original draft: A.S. Writing - review \& editing: A.S, K.S, S.S, N.A. Project administration: A.S. and K.S. Funding acquisition: A.S. and K.S.

\section{Competing interests:}

No competing interests.

Data and materials availability: Data supporting the findings of this study are reported in the main text and figures and in supplementary figs. and tables. Modeling details are reported in Document S1 and the code available for cell counting will be available on GitHub.

\section{Fig. 1: Premolars and molars diversity in noctilionoid bats}

A: Tooth formula and jaw length of noctilionoid bats. Jaw length is represented with a color code and premolar and molars losses events by circles or squares respectively. Some losses happened independently in different clades.

B: Groups of bats made for this study, based on tooth number and jaw length.

$\mathrm{C}$ : Testing the inhibitory cascade model on premolars and molars. Species that follow the IC model colonize the grey triangle. Colors represent jaw length

D: Picture of four species representative of the four different groups representative of jaw size and tooth composition diversity.

Fig. 2: Premolars and molars develop through two different cascades and are lost differently as jaw length decreases in the tree

A: Reconstruction of the developing dental lamina in our four groups of bats on $\mu \mathrm{CT}$ scans. Permanent premolars are indicated in green, deciduous premolars in yellow and molars in pink. 
Premolars and molars develop in two different directions, as the dental lamina grows and matures. Scale bar: $200 \mu \mathrm{m}$

$\mathrm{B}$ : Gradual loss of $\mathrm{dP} 3 / \mathrm{P} 3$ as the relative jaw length decreases. $\mu \mathrm{CT}$ slides of the different premolars are represented around CS23 for the different groups. Species represented in these CTscan are in bold, others can be found in Sup. Fig. 6. Scale bar: $100 \mu \mathrm{m}$

Fig. 3: Growth variation in the premolar region

A: Dynamics of apparition of premolar and molar buds and differential growth, based on $\mu \mathrm{CT}$ scan measurements and cell division experiments. Dental lamina develops in both directions from the initial dP4 and M1 tooth buds independently for premolars and molars. Differential growth rate for the different groups is indicated in green.

B: Growth rate between the Canine (C) and the P4 position during development for species in our four groups calculated from the $\mu \mathrm{CT}$ scans.

$\mathrm{C}$ : Cell division in the premolar and molar regions at relevant stages for Glossophaga soricina (GS), Erophylla sezekorni (ES) and Artibeus jamaicensis (AJ) labelled by EdU in whole mount dissected jaw. The position of the canine is indicated with a white dotted line. CS: Carnegie Stage. Scale bar: $200 \mu \mathrm{m}$

D: Cell division in the premolar and molar regions at relevant stages for Glossophaga soricina (GS), Monophyllus redmani (MR), Mormoops blainvillei (MB) and Artibeus jamaicensis (AJ) labelled by PCNA on cryosections. The position of the canine is indicated with a white dotted line. CS: Carnegie Stage. Scale bar: $250 \mu \mathrm{m}$

Fig. 4: Modelling growth rate in different species is sufficient to recapitulate the variation in tooth number and size observed in Noctilionid bats

A: Reconstruction of the developmental sequences for 3P3MR, 3P3ML and 2P3MS bats using Turing patterns incorporating differential growth. Inhibitory fields are represented by the circles.

B: Reconstruction of the developmental sequence of Artibeus jamaicensis and it tooth size variation using Turing patterns with differential growth. Inhibitory fields are represented by the circles.

C: Modeling the number of premolars and molars by modulating growth rate during the evolution of noctilionoid bats. Adult morphologies are represented on the simplified noctilionoid tree.

\section{Supplementary Material Material and Methods}

Bat groups

We grouped species in 6 morphogroups representative of their dental diversity (Sup Fig 2) relative to tooth number and jaw size. 3P3MR represents the ancestral pattern of 3 premolars and 3 molars and a regular jaw length. 3P3ML represents bats with 3 premolars and 3 molars with elongated jaws as seen mostly in nectar feeders. 2P3MR represents bats with 2 premolars and 3 molars with a shorter jaw length and 2P3MS and 2P2MS represents bats with 2 premolars and 2 or 3 molars, with a short face and jaw. The M3, present in 2P3MS is extremely reduced. In our analyses, because the size of the jaw is an important feature of short-faced stenodermatinea bats, we grouped 2P2MS and 2P3MS bats. In our data set, we also found two species with three premolars and two molars, 3P2M. In these species, the P3 is extremely reduced and their jaw size is closer to the second group. Because this morphology is marginal, it has not been used for 
the following analyses but was kept for the modelling. Finally, we excluded the vampire bats from our analysis given the lack of embryos and their extremely reduced dentition that limit our developmental investigations. Diet groups were assessed based on (24).

\section{Museum and Field specimens}

Museum specimens pictures have been: 1) taken from the FMNH in Chicago with a Nikon camera, 2) downloaded from Animal Diversity Web https://animaldiversity.org/ (see Table S2). Field specimens were collected in the field (see Table S2) using mist nets, harp traps or butterfly nets and euthanized humanely with isoflurane according to approved institutional animal care and use committee (IACUC) protocols 14199 at UIUC, 2017-093 at UCLA and the following permits Dominican Republic: VAPB-01436; Puerto Rico: 2015-EPE-028; Trinidad: 000619 and 000620 Apr 18th 2018. Specimens were then fixed ON at 4C with PFA and dehydrated the next day in $100 \%$ methanol and stored at $-20 \mathrm{C}$ until used.

\section{Body mass}

Body mass was used to normalize the data in our analysis. Body mass data has been collected from Davalos and Melo, Chapter 8, (13), which gathered an impressive dataset on the body size of Noctilionoidea with the exception of: Chilonycteris macleayii=Ptenoronus macleayii (29); Leptonycteris nivalis (30); Lonchophylla thomasii (Emmons, 1990); Platyrrhinus fusciventris, (31).

\section{Statistical analysis}

Differences between the tooth areas of the different groups and teeth were compared by ANOVA and Tukey multiple comparison of means (Table S1 and S3) in R.

\section{Developmental stages}

Developmental stages for the different species of bats have been based on the development of Carollia perspicillata (18).

\section{$\mu C T$ scanning and dental lamina segmentation}

Bat embryo jaws were dissected and stained in $0.3 \%$ (Phosphotungstenic acid, Sigma) PTA in $70 \%$ ethanol (museum specimens) or $100 \%$ methanol (field samples) for 24 to $36 \mathrm{~h}$ on a rocker at room temperature. Stained specimens were mounted in a 1.5 or $2 \mathrm{~mL}$ eppendorf tube between two pieces of foam and $\mu \mathrm{CT}$ scanned in a Skyscan 1172, Scanco uct50 and a Xradia BioMicroCT. Scan parameters were adjusted depending on specimen size and morphology, and voxel size ranged from 1 to $5 \mu \mathrm{m}$ per scan. Raw $\mu \mathrm{CT}$-scan shadow images were reconstructed to slices in NRecon, then imported into Mimics, where the dental lamina was segmented using the lasso tool every 4 to 5 slides before using the interpolation tool. Surface (stl) files were exported and used for visualization and morphological comparisons.

\section{Dental measurements}

Adult dental measurements were taken from museum specimens photos (see Table S2 for the specimens list) or Animal Diversity Web (animaldiversity.org) photo using ImageJ. Scale was set using the scale bar on the pictures. Crown width and length were measured three times for each tooth to ensure reproducibility. For analysis, individuals from the same species and locality were aggregated. Data available upon request. 
Embryo dental measurements were taken from reconstructed .stl files in mimics using the measurement tool. Each distance was measured three times, to ensure reproducibility, between the primary enamel knots (or its resulting cusp) or the tooth primary enamel knot and the end of the dental lamina.

HCR-IHC imaging

Field sampled embryos were embedded in OCT and sectioned using a cryostat CM1520. Proliferated cells were detected using a PCNA antibody (Rabbit mAB \#13110, Cell Signaling technology) at 1:300 and the signal amplified using the HCR system from Molecular Instrument according to the manufacturer protocol (32). Sections were imaged using a Leica SP8 confocal.

\section{EdU staining}

Pregnant females were injected in the field with $20 \mathrm{mg} / \mathrm{kg}$ of EdU reconstituted in DMSO and PBS in an intraperitoneal injection according to IUIAC procedures. 45 minutes after the injection, bats were euthanized with isoflurane. Embryos were dissected out of the female and the jaws were carefully dissected and fixed overnight in 4\% PFA at 4C before being dehydrated the next day in $100 \%$ methanol and stored at -20C until imaging. In the lab, half dissected jaws were rehydrated and clarified using Scale S (33). After the incubation in the S3 reagent, jaws were re-hydrated in PBS before labelling. The next day was stained with a Click-iT EdU Alexa fluor 647 labelling kit according to the manufacturer protocol except for the incubation time adjusted to $3 \mathrm{~h}$ ar RT. After the reaction, jaws were put in Scale S4 for the final clarification and imaging at the Leica SP8 DIVE two-photon microscope at the CNSI facility at UCLA. Resulting photos were processed using LASX and ImageJ.

\section{EdU cell counting}

To count the nucleus in 3D reconstructed images (Fig. S7) from the two-photon, we used a script in ImageJ (code available upon request). The spot counter is based on Gaussian blurring, maximum detection and filtering maxima above a given threshold. The Gaussian blur ensures that only one maximum per nucleus is found. The threshold allows to exclude maxima in the background and is adapted to each image to ensure that each nucleus is counted once. Each image is divided in squares of $128 \times 128$ pixels and nucleus counted in each squares.

\section{Modeling}

See Supplementary Document 1.

Fig. S1: Tooth and jaw morphology of the different bats

Phylogeny of all the species used in our dataset, their dental formula and the seven initial groups based on jaw length and tooth number (see Table 1). For each species, the main diet is indicated. The events of premolar and molar gains and losses are also indicated on the tree.

Fig. S2: Representative bats from the different groups based of tooth number and jaw length Pictures from ADW (animaldiversity.org, Phil Myer).

Fig. S3: Tooth proportion variation in the different groups of bats

Tooth proportions variation in the different groups of bats (see Methods). As jaw length diminishes, molar proportions become less equal, in particular for 2P3MS. Most of the variation 
in terms of M1 and M2 proportions in all bats is explained by the 2P2MS group. Tooth sizes are normalized by adult body mass.

Fig. S4: Relation between tooth number and jaw length

A: Tooth number is correlated with postcanine dental field length. B: Dental field length variation in the different groups. C: Jaw length variation in the different groups.

Fig. S5: Classical single DIC hypothesis for the P3-P4-M1triplet

Testing the inhibitory cascade on the triplets P4-M1-M2 which would imply a cascade starting at P4 and then going in both directions. The high value of the ratio reflects the differences in size between molars and premolars. Colors: groups identified in Fig. 1.

Fig. S6: $\mu \mathrm{CT}$ scan on isolated stages for more species

Reconstruction of the developing dental lamina in three other species of bats for which the developmental series is incomplete. Permanent premolars are indicated in green, deciduous premolars in yellow and molars in pink. For E. sezekorni, the dP3 is indicated with a yellow arrow and will give rise to a functional dP3. For $U$. bilobatum and A. phaeotis, the developing $\mathrm{dP} 3$ is indicated with a yellow arrow at its earlier stages but then degenerates before its eruption in the juveniles.

Fig. S7: EdU nucleus counting from two-photon imaging

Density maps (left) of nucleus in 3D reconstructed images (right) were reconstructed using a script in ImageJ (code available upon request).

A: Density maps of the species presented in Fig. 3 (GS CS22: G. soricina, carnegie stage 22; ES CS21: E. sezekorni carnegie stage 21, AJ CS19: A. jamaicensis, carnegie stage 19).

B: Density maps of additional species (GS CS20.5: G. soricina, carnegie stage 20.5; AJ CS23: A. jamaicensis, carnegie stage 23).

Square size: $128 \times 128 p x$

Table S1: $\underline{\text { Size of the jaw and dental formula }}$

A: ANOVA comparing jaw length, between the 5 different dental formula groups

$\mathrm{B}$ : Tukey multiple comparisons of means. 2P2MS jaws are significantly different from 3P3MR and $3 \mathrm{P} 2 \mathrm{M}$. 3P3ML is significantly different from 2P3MR. 3P3MR and 3P3ML are significantly different from 2P3MS.

Table S2: Specimen list

Table S3: Mean comparison between teeth

ANOVA and Tukey multiple comparisons of means between tooth proportions for the different categories.

Document S1: Modeling 
bioRxiv preprint doi: https://doi.org/10.1101/2021.12.05.471324; this mersion posted December 7, 2021. The copyright holder for this preprint (which was not certified by peer review) is the althor/funder. All rights reserved. No reuse allowed without permission.
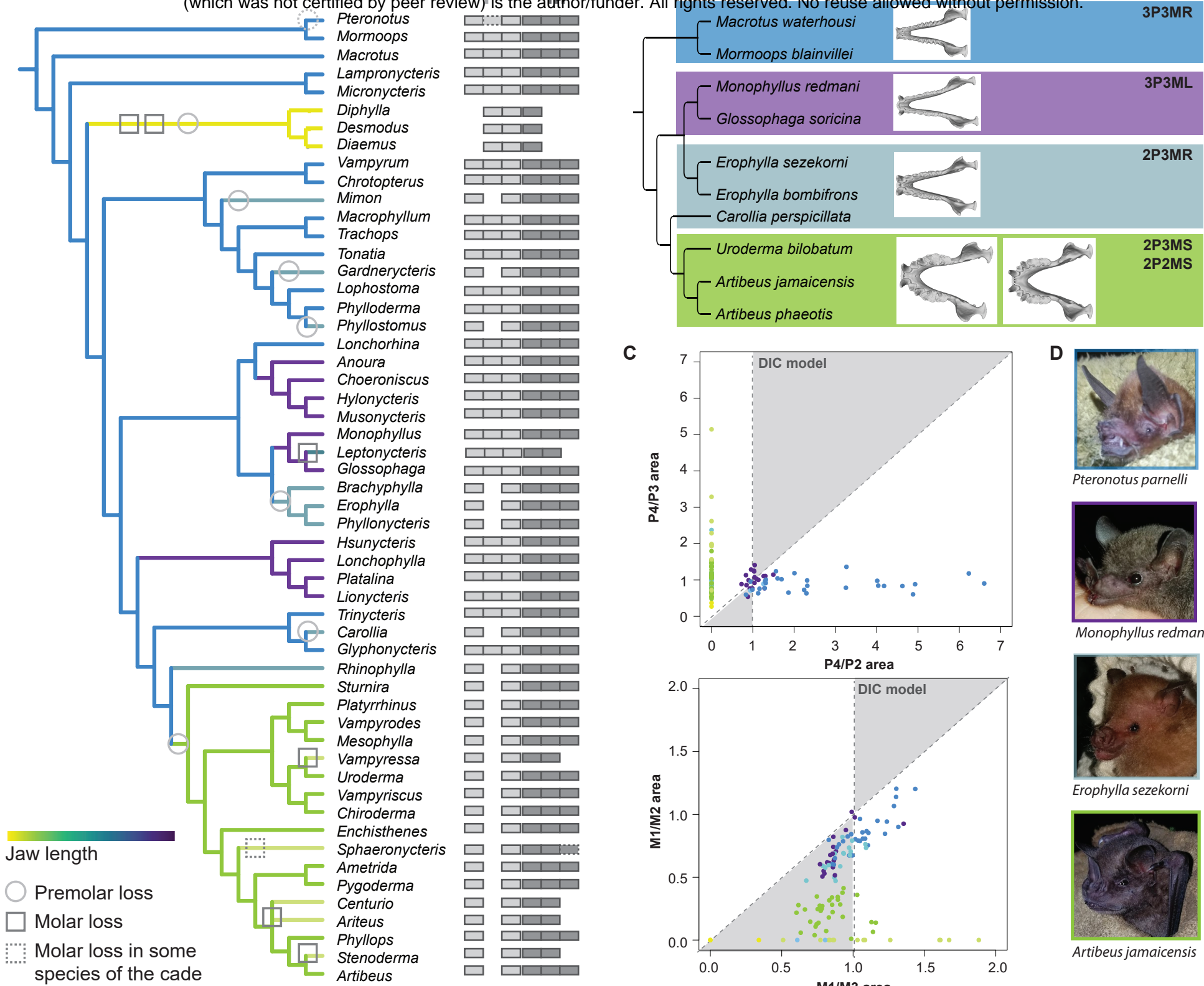

C
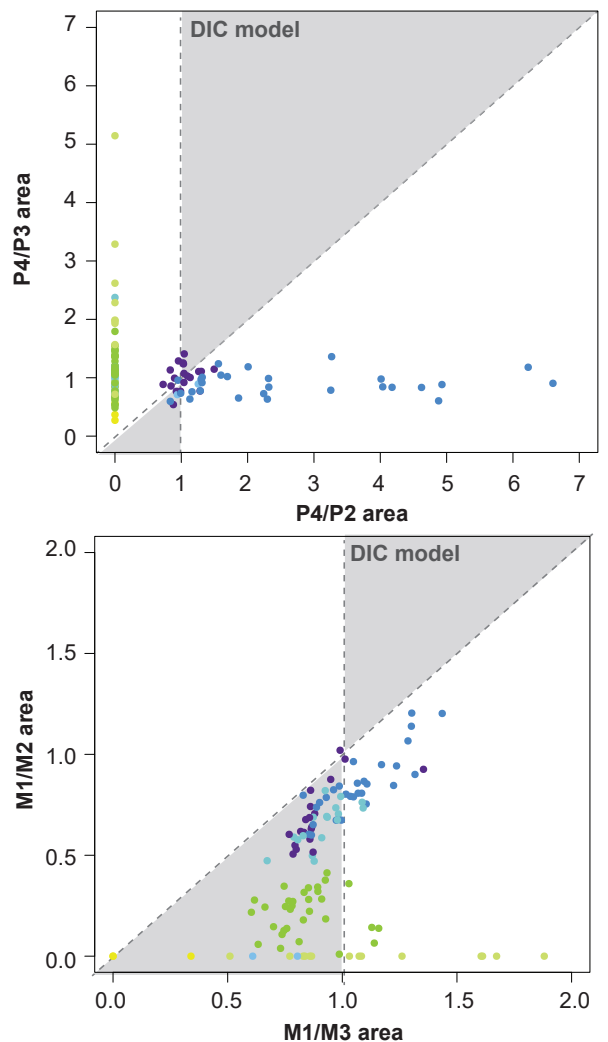

D
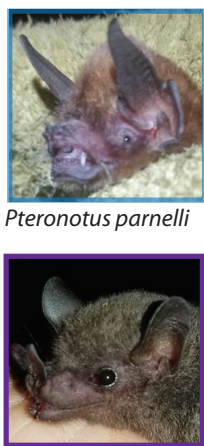

Monophyllus redmani

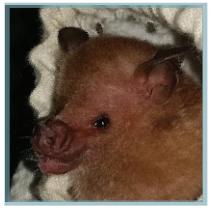

Erophylla sezekorni

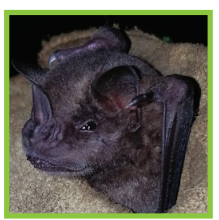

Artibeus jamaicensis

\section{Figure 1}




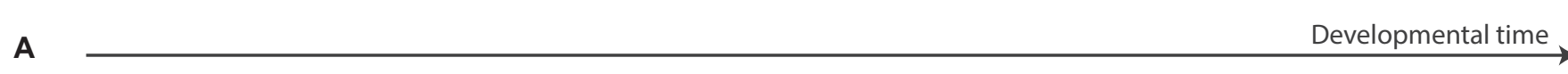

A

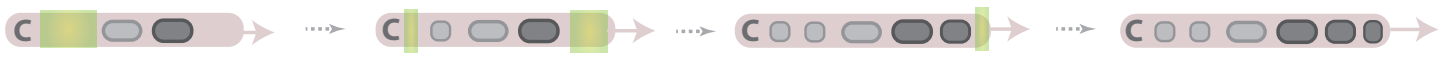

3P3MR

bioRxiv preprint doi: https://doi.org/10.1101/2021.12.05.471324; this version posted December 7, 2021. The copyright holder for this preprint (which was not certified by peek review) is the author/funder. All rights reserved_-No reuse allewed without permission.

3P3ML

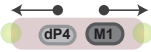

$P \underset{\text { La }}{\stackrel{L i}{L} D}$
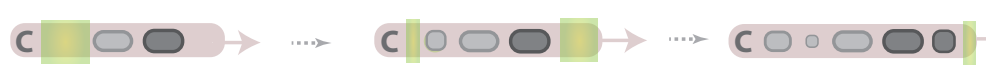

c $\bigcirc \circ \bigcirc \bigcirc 00$

2P3MS

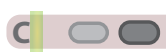

c $0 \bigcirc \bigcirc$

c $\bigcirc \circ \circ$

c 0000

B

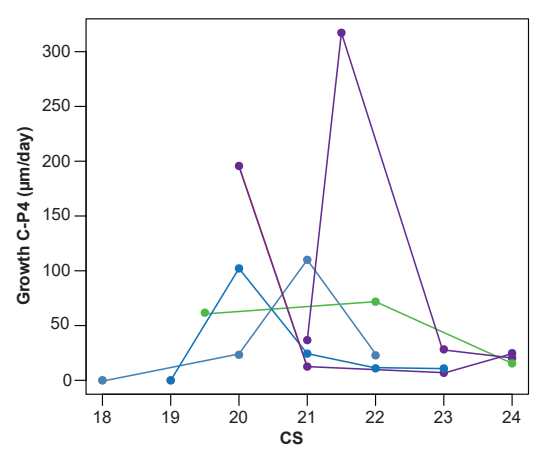

C

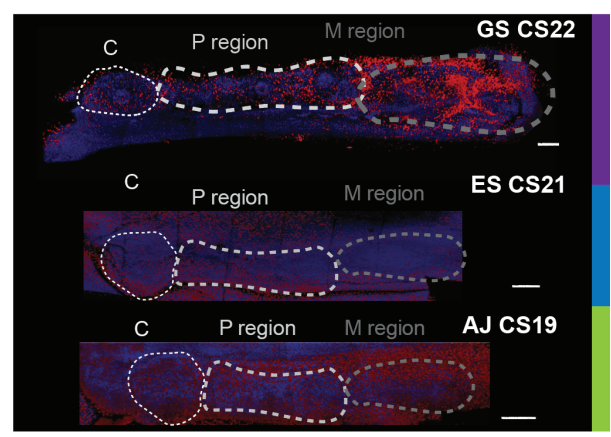

D

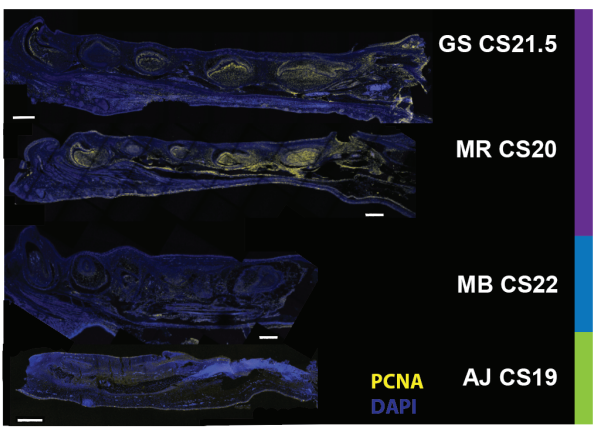

Figure 3 
bioRxiv preprint doi: https://doi.org/10.1101/2021.12.05.471324; this version posted December 7, 2021. The copyright holder for this preprint P4 W

\section{- • • •}

$\cdot \cdot \cdot \cdot \cdot$

• $\bullet \cdot 1 \cdot 0 \cdot$

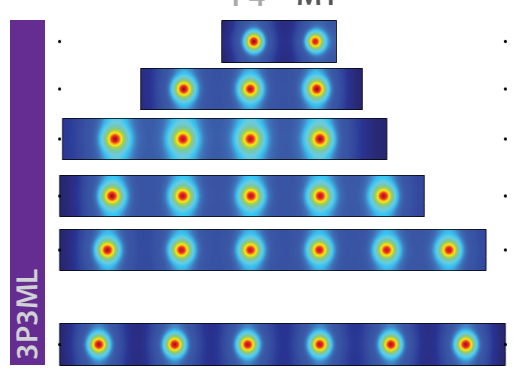

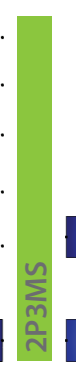

c

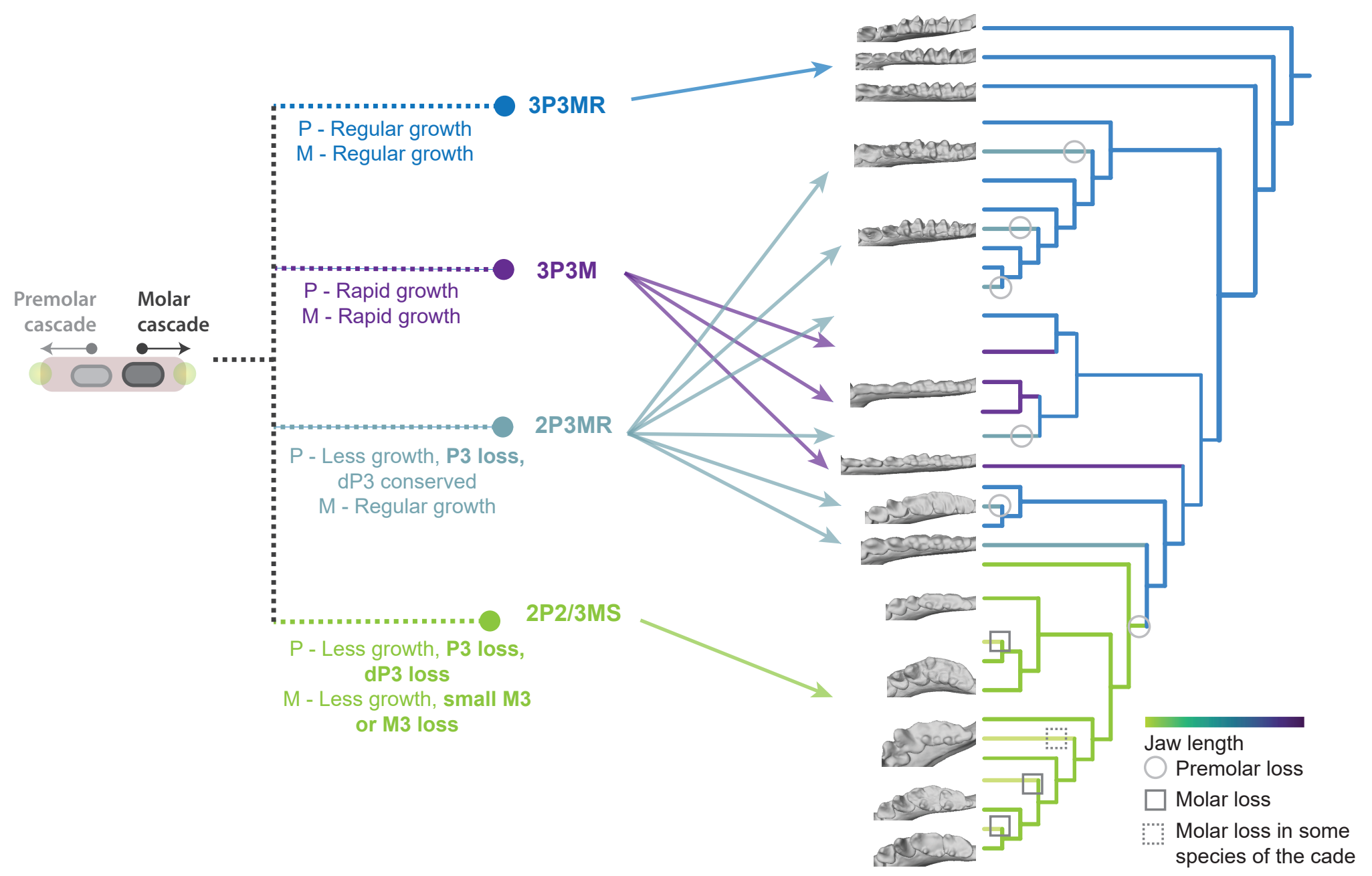

Figure 4 